\title{
Coherent Photon States and Spectral Condition
}

\author{
G. ROEPSTORFF \\ II. Institut für Theoretische Physik der Universität Hamburg \\ Received July 1, 1970
}

\begin{abstract}
A criterion is derived for the existence of a selfadjoint and semibounded momentum operator in a non-Fock representation of the free photon field given by a coherent state. The representation of the translation group is constructed and it is shown that the rotation group, hence the homogeneous Lorentz group, cannot be unitarily implemented in the so-called infrared sectors.
\end{abstract}

\section{Introduction}

It has been stated many times that the theory of soft photon emission primarily deals with non-Fock representations of the outgoing free electromagnetic field. As is well known, the use of strange, so-called infrared representations is necessitated by the singular behavior of the convection current of the accelerated charged particle at the vertex of the light cone in momentum space [1]. It has further been realized that coherent states $[2,3]$, suitably generalized to account for an "infinite photon number", not only provide a useful tool to cope with infrared problems in quantum electrodynamics [4-7], but also arise naturally in a study of classical external currents $[8,9]$, the Bloch-Nordsieck [10] and the Pauli-Fierz model $[11,12]$. A deeper reason for the occurrence of coherent states may be seen in their intimate connection with the Poisson process which governs the photon emission.

Any coherent state, considered as a functional on the quantized radiation field, gives rise to a representation of that field and, clearly, the existence of a momentum operator in that representation which is inevitable for a spectral analysis of the photon emission restricts the class of admissible states. Therefore, we aim to find a necessary and sufficient condition for a coherent state to define a sector that is covariant with respect to the space-time translations ${ }^{1}$. Assuming this condition to be satisfied, it will become evident that in general the energy is not bounded from below and, as a consequence, cannot be uniquely defined. A second criterion then assures that a momentum operator with physical spectrum

1 We mention that our discussion will have various points of contact with the theory of direct product representations [13], exponential Hilbert spaces [14], and infinitely divisible group representations [15], but will not draw on any results of these works. 
exists for a subclass of states. These states then give rise to the infrared sectors that do not admit a number operator and it will be seen that not even an angular momentum operator exists for these sectors, since the rotation group cannot be implemented.

The degrees of freedom for the electromagnetic radiation are conveniently described by a vector field $A(\varphi)=\int d x \varphi_{\mu}(x) A^{\mu}(x)$. We take the view that the potential $A^{\mu}(x)$ is neither unique nor observable, but the field strengths $\partial^{\mu} A^{v}-\partial^{v} A^{\mu 2}$ are. Therefore and in accord with the local nature of all measurements, we limit ourselves to test functions $\varphi_{\mu}=\partial^{v} \varphi_{\mu v}$, where $\varphi_{\mu \nu}$ is real, antisymmetric, has compact support and partial derivatives of any order. Let $D$ be the singular commutator function. Then the convolution product

$$
f_{\mu \nu}=D *\left(\partial_{\mu} \varphi_{\nu}-\partial_{\nu} \varphi_{\mu}\right)
$$

maps our test functions onto a set $L$ of smooth solutions of the wave equation. Relative to the usual definitions of addition and scalar multiplication of functions, $L$ is a real-linear vector space.

We shall mainly work with the bounded Weyl operators $W(f)$ $=\exp i A(\varphi)$, written as a function of $f \in L$, on which $A(\varphi)$ naturally depends. As a substitute for the commutation relations of the fields we find the multiplication law

$$
W\left(f_{1}\right) W\left(f_{2}\right)=W\left(f_{1}+f_{2}\right) e^{-i \sigma\left(f_{1}, f_{2}\right)}
$$

$\sigma$ being a regular symplectic form on $L$ defined $^{3}$ by

$$
\sigma\left(f_{1}, f_{2}\right)=\frac{1}{2} \int d x \varphi_{1}{ }^{\mu}(x) D(x-y) \varphi_{2 \mu}(y) .
$$

Certain topologies on $L$ will be of interest to us. With aid of the Fourier transform $\tilde{\varphi}_{\mu}(k)=\int d x \varphi_{\mu}(x)$ expikx we introduce a decreasing sequence of pairwise compatible norms $\|f\|_{n>-4}$ on $L$ setting ${ }^{4}$

$$
\|f\|_{n}^{2}=(2 \pi)^{-3} \int \frac{d \boldsymbol{k}}{2|\boldsymbol{k}|}\left(\frac{\boldsymbol{k}^{2}}{1+\boldsymbol{k}^{2}}\right)^{\frac{n}{2}} \sum_{i=1}^{3}\left|\tilde{\varphi}_{i}(k)-\frac{k_{i}}{k_{0}} \tilde{\varphi}_{0}(k)\right|_{k_{0}=|\boldsymbol{k}|}^{2} .
$$

The completion of $L$ relative to the norm $\|f\|_{n}$ yields a complex Hilbert space $L_{n}$, in which the multiplication of $f \in L$ by the imaginary unit is formally effected if $D$ in (1.1) is replaced by the acausal function $D^{(1)}$.

2 The notion is $\partial^{\mu}=\partial / \partial x_{\mu}$ and $x=\left\{x_{\mu}\right\}=\left\{x_{0}, \boldsymbol{x}\right\}$. We use the inner product $k x=k_{0} x_{0}-\boldsymbol{k} \cdot \boldsymbol{x}$ and choose $d \boldsymbol{x}$ and $d x$ to denote the Lebesgue measures on $\boldsymbol{R}^{3}$ and $\boldsymbol{R}^{4}$ respectively.

${ }^{3}$ Since $\sigma\left(f_{1}, f_{2}\right)=\frac{1}{4} \int d x f_{1}^{\mu \nu}(x) \varphi_{2 \mu \nu}(x)=-\frac{1}{4} \int d x \varphi_{1}^{\mu v}(x) f_{2 \mu \nu}(x)$, it is apparent that $\sigma\left(f_{1}, f_{2}\right)$ does not depend on the particular choice of $\varphi_{1}$ and $\varphi_{2}$, but merely on $f_{1}$ and $f_{2}$.

${ }_{4}$ Note that $|\boldsymbol{k}|^{-1} \tilde{\varphi}_{\mu}(|\boldsymbol{k}|, \boldsymbol{k})$ is bounded at $\boldsymbol{k}=0$, for $\tilde{\varphi}_{\mu}(k)$ is an entire function satisfying $\tilde{\varphi}_{\mu}(0)=0$. 
Clearly, if $\notin L$ in general if $f \in L$. The space $L_{n}$ may be identified with a dense subspace in $L_{m}$ if $n<m$ and, with respect to the inner product in $L_{0}, L_{n}$ may also be identified with $L_{-n}^{\prime}$, the strong dual of $L_{-n}$. The inner product for $L_{n}$, as derived from (1.4), shall be denoted by $(f, g)_{n}$. A simple calculation shows that $\sigma(f, g)=\operatorname{Im}(f, g)_{0}$ if $f, g \in L$.

\section{Weyl Systems and Coherent States}

A Weyl system $(\mathfrak{H}, W)$ over the real symplectic space $(L, \sigma)$ consists of a Hilbert space $\mathfrak{H}$ and a map $f \mapsto W(f)$ of $L$ into $\mathfrak{U}(\mathfrak{H})$, the set of unitary operators on $\mathfrak{H}$, such that the Weyl relations (1.2) hold, $W(0)=I$ (unit), and such that for each $f \in L$ the map $\lambda \mapsto W(\lambda f), \boldsymbol{R} \rightarrow \mathfrak{U}(\mathfrak{H})$ is continuous with respect to the weak operator topology. We note that $W(f)^{*}$ $=W(-f)$. A cyclic Weyl system $(\mathfrak{H}, W, \Omega)$ is a Weyl system admitting a cyclic vector $\Omega \in \mathfrak{H}$. By a state (more precisely, a regular state) we shall mean a functional $E: L \rightarrow C$ satisfying $E(0)=1$ and $\sum_{j, k} \bar{c}_{j} c_{k} E\left(f_{k}-f_{j}\right)$ - $\exp i \sigma\left(f_{j}, f_{k}\right) \geqq 0$, for which $\lambda \mapsto E(f+\lambda g), \boldsymbol{R} \rightarrow \boldsymbol{C}$ is continuous. An extreme point of the convex set of all states is called a pure state.

Given a Weyl system $(\mathfrak{H}, W)$ and a unit vector $\Omega \in \mathfrak{H}$, then $E(f)$ $=(\Omega, W(f) \Omega)$ defines a state. Conversely, by virtue of the Gel'fand-Segal construction there is, for any state $E$, a cyclic Weyl system $(\mathfrak{H}, W, \Omega)$ such that $(\Omega, W(f) \Omega)=E(f)$. Assume $E$ is pure and let $\Phi$ be any unit vector in $\mathfrak{H}$, then $E_{\Phi}(f)=(\Phi, W(f) \Phi)$ again defines a pure state. The set of these states will be called the sector associated with $E$. For a short account of the algebraic concepts, the reader is referred to [16]. Weyl systems over a real symplectic space have been discussed by many authors [17-21].

Among all states of interest, the vacuum state $E_{0}(f)=\exp \left\{-\frac{1}{2}\|f\|_{0}^{2}\right\}$ plays a dominant part. By way of the GS construction we obtain a cyclic Weyl system $\left(\mathfrak{G}_{0}, W_{0}, \Omega\right)$ with $E_{0}(f)=\left(\Omega, W_{0}(f) \Omega\right)$. To abbreviate, we shall refer to $\left(\mathfrak{H}_{0}, W_{0}, \Omega\right)$ as the Fock system. Three simple facts have been repeatedly proved in the literature in different contexts:

(1) $E_{0}$ is a pure state and, as a consequence, $W_{0}$ is irreducible.

(2) $f \mapsto W_{0}(f)$ is continuous with respect to the strong operator topology for $\mathfrak{U}\left(\mathfrak{S}_{0}\right)$ and the $L_{0}$ norm topology for $L$. Thus, $W_{0}$ has a unique extension to a continuous map $L_{0} \rightarrow \mathfrak{U}\left(\mathfrak{H}_{0}\right)$ (which we shall again denote by $\left.W_{0}\right)$ and $W_{0}(\xi) W_{0}(\eta)=W_{0}(\xi+\eta) \exp \left\{-i \operatorname{Im}(\xi, \eta)_{0}\right\}$ for all $\xi, \eta \in L_{0}$.

(3) The Fock system admits a number operator $N$. For, by linearity and continuity, the map $W_{0}(f) \Omega \mapsto W_{0}\left(e^{i t} f\right) \Omega$ can be extended to a unitary operator $e^{i N t}$, the significance of $\|f\|_{0}^{2}$ being that it gives the mean number of photons in the state induced by the vector $W_{0}(f) \Omega$. 
Let $L^{\prime}$ be the algebraic dual of $L$, i.e. the set of real-linear functionals $F: L \rightarrow \boldsymbol{R}$. Given $F \in L^{\prime}$, the definition

$$
E_{F}(f)=E_{0}(f) e^{i F(f)}
$$

yields what will be called a coherent state. Setting

$$
W_{F}(f)=W_{0}(f) e^{i F(f)}
$$

we obtain a cyclic Weyl system $\left(\mathfrak{G}_{0}, W_{F}, \Omega\right)$ for which

$$
\left(\Omega, W_{F}(f) \Omega\right)=E_{F}(f) \text {. }
$$

Therefore, the system $\left(\mathfrak{H}_{0}, W_{F}, \Omega\right)$ is unitarily equivalent to the cyclic system canonically associated with the state $E_{F}$. Automorphisms of the form (2.2) are extremely useful in order to obtain new sectors from known ones. States are thus not characterized by specifying a vector in $\mathfrak{S}_{0}$, but by specifying both the vector and the representation.

The proof of the following lemma is adapted from Manuceau [21, 22].

Lemma 1. Given $F_{1}, F_{2} \in L^{\prime}$, the states $E_{F_{1}}$ and $E_{F_{2}}$ belong to the same sector if and only if $\left|F_{1}(f)-F_{2}(f)\right| \leqq C\|f\|_{0}$. In this case there is a unique vector $\xi$ in $L_{0}$ such that $F_{1}(f)-F_{2}(f)=2 \operatorname{Im}(\xi, f)_{0}$. Any intertwining operator, i.e. any operator $A$ on $\mathfrak{S}_{0}$ with $A W_{F_{1}}(f)=W_{F_{2}}(f) A$, is of the form $\lambda W_{0}(\xi), \lambda \in C$.

Proof. Assume $|D(f)| \leqq C\|f\|_{0}$ for $D=F_{1}-F_{2}$. Then $D$ has a unique extension to a continuous real-linear functional $D: L_{0} \rightarrow \boldsymbol{R}$. Obviously, $2 \Delta(f)=D(i f)+i D(f)$ is complex-linear and continuous. By Riesz' representation theorem, $\Delta(f)=(\xi, f)_{0}$ with unique $\xi \in L_{0}$ and thus $D(f)=2 \operatorname{Im}(\xi, f)_{0}$. Further, $\left(\Phi, W_{F_{2}}(f) \Phi\right)=E_{F_{1}}$ if $\Phi=W_{0}(\xi) \Omega$, proving that $E_{F_{1}}$ and $E_{F_{2}}$ belong to the same sector. Conversely, if $E_{F_{1}}$ and $E_{F_{2}}$ are in the same sector, then $U W_{F_{1}}(f)=W_{F_{2}}(f) U$ for some suitable unitary operator $U$ on $\mathfrak{S}_{0}$ and $\exp i D(f)=\left(U W_{0}(f) \Omega, W_{0}(f) U \Omega\right)$. The right hand side is continuous in $f$ relative to the $L_{0}$ norm topology. Suppose $D(f)$ is discontinuous. Taking advantage of the linearity, one may find a Cauchy sequence $f_{n} \in L$ such that $\lim \left\|f_{n}\right\|_{0}=0$ but $\lim D\left(f_{n}\right)=\pi$ and so $\lim \exp i D\left(f_{n}\right)=-1$, contradicting the continuity of $f \mapsto \exp i D(f)$. Thus, the functional $D$, being continuous and linear, is bounded. Finally we observe that $A W_{0}(\xi)^{*}$ commutes with any $W_{0}(f), f \in L$, if $A$ is an intertwining operator, and hence $A=\lambda W_{0}(\xi)$ by Schur's lemma, for $W_{0}$ is irreducible.

\section{Translations and Cocycles}

Let $G$ be the group of space-time translations acting on $L$ in an obvious way: $(x) f_{\mu \nu}(y)=f_{\mu \nu}(y-x)$. Clearly, the map $f \mapsto(x) f$ extends to an unitary operator $V(x)$ on $L_{n}$ and $V$ is a strongly continuous repre- 
sentation of $G^{5}$. Similarly, the map $W_{0}(f) \Omega \mapsto W_{0}((x) f) \Omega$ by way of linearity, can be extended to a linear isometry $U_{0}(x)$ defined on a dense set in $\mathfrak{H}_{0}$. In a second step, using continuity, $U_{0}(x)$ is seen to act as a unitary operator on the whole of $\mathfrak{H}_{0}$. Again, $U_{0}$ is a strongly continuous representation of $G[21,22]$.

Given $F \in L^{\prime}$ and $x \in G$, the map

$$
W_{F}(f) \mapsto W_{F}((x) f)
$$

defines an automorphism of the Weyl system $\left(\mathfrak{H}_{0}, W_{F}, \Omega\right)$. Since $\Omega$ is invariant under $U_{0}(x)$, it is immediate that, if $F$ is also translationally invariant, $U_{0}(x) W_{F}(f)=W_{F}((x) f) U_{0}(x)$, i.e. $U_{0}(x)$ implements the automorphism. However, it fails to implement (3.1) in the general case. There we have:

Proposition 1. The following statements are equivalent:

(1 a) For any $x \in G$, the automorphism (3.1) can be unitarily implemented.

(1b) $|F(f-(x) f)| \leqq C(x)\|f\|_{0}$.

(1 c) $F(f-(x) f)=2 \operatorname{Im}(\xi(x), f)_{0}, \quad \xi(x) \in L_{0}$.

If $U(x) W_{F}(f)=W_{F}((x) f) U(x)$ for some unitary $U(x)$, then

$$
U(x)=\lambda(x) U_{0}(x) W_{0}(\xi(x))
$$

for some complex $\lambda(x)$ of unit modulus. Moreover, the function $\xi(x)$ satisfies:

$$
\xi(x+y)=V(y)^{*} \xi(x)+\xi(y) .
$$

Proof. Setting $F_{x}(f)=F((x) f)$ we get $W_{F}((x) f)=U_{0}(x) W_{F_{x}}(f) U_{0}(x)^{*}$. Hence, (3.1) can be unitarily implemented if and only if the states $E_{F}$ and $E_{F_{x}}$ belong to the same sector. The equivalence of the statements $(1 \mathrm{a}-\mathrm{c})$ then follows from Lemma 1. Suppose $U(x) W_{F}(f)=W_{F}((x) f) U(x)$ for some unitary $U(x)$. Then $U_{0}(x)^{*} U(x) W_{F}(f)=W_{F_{x}}(f) U_{0}(x)^{*} U(x)$ and thus $U_{0}(x)^{*} U(x)=\lambda(x) W_{0}(\xi(x))$ using Lemma 1. Finally,

$$
F(f)-F_{x}(f)=2 \operatorname{Im}(\xi(x), f)_{0}
$$

and

$$
\left(F_{x+y}(f)-F(f)\right)+\left(F((y) f)-F_{x}((y) f)\right)+\left(F(f)-F_{y}(f)\right)=0
$$

imply

$$
\operatorname{Im}\left(-\xi(x+y)+V(y)^{*} \xi(x)+\xi(y), f\right)_{0}=0
$$

for $f \in L$ arbitrary. Since $L$ is dense in $L_{0}$, (3.3) follows. This completes the proof.

${ }^{5}$ Strictly speaking, $V$ depends on $n$. We shall, however, suppress this label, since no confusion seems to be possible. 
Adding one more condition on $\xi(x)$, namely continuity, the function $\xi$, by virtue of the defining Eq. (3.3), will be called a 1-cocycle on $G$ relative to the representation $V$ of $G$ on $L_{0}$. The set of these cocycles is a vector space with respect to the usual definitions of addition and scalar multiplication of functions. Following Araki [23], this space is denoted by $Z^{1}\left(G, L_{0}\right)$. Any constant vector $\eta \in L_{0}$ determines a 1 -cocycle $\xi$ by $\xi(x)=\left(I-V(x)^{*}\right) \eta$, called a 1 -coboundary. The set $B^{1}\left(G, L_{0}\right)$ of these coboundaries is thus a linear subspace of $Z^{1}\left(G, L_{0}\right)$.

If we planned to study a free Bose field of non-vanishing mass, every 1-cocycle would be a 1-coboundary due to the different spectral properties of the representation $V$ of $G$. However, this is no longer true in the case of mass-zero fields, a complication which is responsible for the occurrence of so-called infrared representations. In any case, the main question is, whether it is possible to characterize the quotient space

$$
H^{1}\left(G, L_{0}\right)=Z^{1}\left(G, L_{0}\right) / B^{1}\left(G, L_{0}\right) .
$$

At this point of the discussion one realizes the significance of the spaces $L_{n}$ defined in the Introduction. Note that

$$
K(x)^{2}=\sup _{\boldsymbol{k}} \frac{1+\boldsymbol{k}^{2}}{\boldsymbol{k}^{2}}\left|1-e^{-i k x}\right|_{k_{0}=|\boldsymbol{k}|}^{2}
$$

is finite for any $x \in G$ and $\left\|\left(I-V(x)^{*}\right) f\right\|_{n} \leqq K(x)\|f\|_{n+2}$. Hence,

$$
\left(I-V(x)^{*}\right) L_{n+2} \subset L_{n}
$$

holds for any $x \in G$. In particular, the function $\xi(x)=\left(I-V(x)^{*}\right) \eta$ is seen to be an element of $Z^{1}\left(G, L_{0}\right)$ if $\eta \in L_{2}$. The linear space of these 1-cocycles will be referred to as $B^{1}\left(G, L_{2}\right)$. As would be expected intuitively, these cocycles exhaust $Z^{1}\left(G, L_{0}\right)$ :

Lemma 2. $Z^{1}\left(G, L_{0}\right)=B^{1}\left(G, L_{2}\right)$ and thus $H^{1}\left(G, L_{0}\right)$ may be identified with $L_{2} / L_{0}$.

We shall not attempt to prove this lemma, but remark that $V$ does not contain the trivial representation of $G$. The lemma then follows from a general result ${ }^{6}$ of Araki [23, Lemma 7.2].

Proposition 2. The following statements are equivalent:

(2a) There exists a continuous projective representation of $G$ on $\mathfrak{H}_{0}$ that implements the automorphisms (3.1).

${ }^{6}$ In order to establish the connection with Araki's work, the function $h(t)$ used in Definition 6.1 of [23] may be chosen to be $\frac{1}{2} e^{-|t|}$ yielding $\tilde{h}(\lambda)=\left(1+\lambda^{2}\right)^{-1}$. Then $\sum_{\mu=0}^{3}\left(1-\tilde{h}\left(k_{\mu}\right)\right)=\Phi(k) \boldsymbol{k}^{2}\left(1+\boldsymbol{k}^{2}\right)^{-1}$ and $2 \leqq \Phi(|\boldsymbol{k}|, \boldsymbol{k}) \leqq 4$ may be used to show that the space $L_{2}$ coincides with the space $\bar{D}^{+}$in Araki's notation. 
(2b) A function $C(x)$ on $G$ exists such that $\lim _{x \rightarrow 0} C(x)=0$ and

$$
|F(f-(x) f)| \leqq C(x)\|f\|_{0} .
$$

(2c) $F$ admits a unique decomposition $F^{(1)}+F^{(2)}$, where $F^{(1)}$ is translationally invariant and $F^{(2)}(f)=2 \operatorname{Im}(\eta, f)_{0}$ for some $\eta \in L_{2}$.

Moreover, the projective representation in (2a) can be replaced by a strongly continuous unitary representation.

Proof. $(2 \mathrm{a}) \Rightarrow(2 \mathrm{~b}) \Rightarrow(2 \mathrm{c})$ : Suppose $U(x) W_{F}(f)=W_{F}((x) f) U(x)$, where $U(x)$ is unitary for each $x \in G$ and $x \mapsto|(\Omega, U(x) \Omega)|$ is continuous. By Proposition 1, $F(f-(x) f)=2 \operatorname{Im}(\xi(x), f)_{0}$ and, using (3.2),

$$
\|\xi(x)-\xi(y)\|_{0}^{2}=-2 \log |(\Omega, U(x-y) \Omega)|,
$$

so that $\xi(x)$ is continuous. With $C(x)=2\|\xi(x)\|_{0}$, the property (2b) follows. Being a 1-cocycle, $\xi(x)$ may, according to Lemma 2, be written as $\xi(x)=\left(I-V(x)^{*}\right) \eta$ for some $\eta \in L_{2}$, implying that $F^{(1)}(f)=F(f)$ $-2 \operatorname{Im}(\eta, f)_{0}$ is translationally invariant.

$(2 \mathrm{c}) \Rightarrow(2 \mathrm{~b})$ : Use $C(x)=2\left\|\left(I-V(x)^{*}\right) \eta\right\|_{0}$.

$(2 \mathrm{c}) \Rightarrow(2 \mathrm{a})$ : Use $U(x)=U_{0}(x) W_{0}(\xi(x)), \xi(x)=\left(I-V(x)^{*}\right) \eta$, to implement the automorphisms (3.1). Note that $x \mapsto W_{0}(\xi(x))$ as a composition of continuous maps is continuous.

The last part of the proposition will follow from a structure analysis of $U(x)=U_{0}(x) W_{0}(\xi(x))$ with $\xi \in Z^{1}\left(G, L_{0}\right)$. We get

$$
\begin{aligned}
U(x) U(y) & =e^{i \hat{\xi}(x, y)} U(x+y), \\
\hat{\xi}(x, y) & =\operatorname{Im}(\xi(x), \xi(-y))_{0}
\end{aligned}
$$

using $W_{0}(\xi(x)) U_{0}(y)=U_{0}(y) W_{0}\left(V(y)^{*} \xi(x)\right)$, the Weyl relations, (3.3), and the identity $V(y) \xi(y)=-\xi(-y)$ which follows from (3.3). Clearly, the real function $\hat{\xi}(x, y)$ is continuous in each variable, it satisfies the relation $\hat{\xi}(x, y)+\hat{\xi}(x+y, z)=\hat{\xi}(y, z)+\hat{\xi}(x, y+z)$ and is thus an element of $Z^{2}(G, R)$, the space of real-valued 2-cocycles on $G$. Suppose, there is some real continuous function $\zeta(x)$ with $\zeta(x+y)-\zeta(x)-\zeta(y)=\hat{\xi}(x, y)$, then $\hat{\xi}$ is called a 2 -coboundary, i.e. $\hat{\xi} \in B^{2}(G, \boldsymbol{R})$, and

$$
\begin{aligned}
U_{\eta}(x) & =e^{i \zeta(x)} U_{0}(x) W_{0}(\xi(x)), \\
\xi(x) & =\left(I-V(x)^{*}\right) \eta
\end{aligned}
$$

defines a strongly continuous unitary representation $U_{\eta}$ of $G$ on $\mathfrak{H}_{0}$. It is therefore of vital importance to realize that any 2-cocycle defined by (3.5) is indeed a 2-coboundary.

Lemma 3. For any $\xi \in Z^{1}\left(G, L_{0}\right)$ there is a finite positive Borel measure $\mu$ on $\boldsymbol{R}^{3}$ which is absolutely continuous relative to Lebesgue measure and which satisfies

$$
(\xi(x), \xi(-y))_{0}=\int d \mu(\boldsymbol{k}) \frac{1+\boldsymbol{k}^{2}}{\boldsymbol{k}^{2}}\left(e^{i k x}-1\right)\left(e^{i k y}-1\right)_{k_{0}=|\boldsymbol{k}|} .
$$


The equation $\zeta(x+y)-\zeta(x)-\zeta(y)=\operatorname{Im}(\xi(x), \xi(-y))_{0}$ has a solution $\zeta$ and any real continuous solution can be written

$$
\zeta(x)=q x+\int d \mu(\boldsymbol{k}) \frac{1+\boldsymbol{k}^{2}}{\boldsymbol{k}^{2}}\left[\sin k x-\frac{k x}{\left(1+\boldsymbol{k}^{2}\right)^{\frac{1}{2}}}\right]_{k_{0}=|\boldsymbol{k}|}
$$

where $q \in \boldsymbol{R}^{4}$ is arbitrary.

Proof. Being a 1-cocycle, $\xi(x)$ may be written as $\left(I-V(x)^{*}\right) \eta$ with $\eta \in L_{2}$. Then $(\eta, V(x) \eta)_{2}=\int d \mu(\boldsymbol{k})(\exp i k x)_{k_{0}=|\boldsymbol{k}|}$ determines $\mu$ with the required properties. It is further evident that the integral in (3.7) converges and, as a function of $x$, has a 2-coboundary which coincides with $\hat{\xi}$. Given two real solutions $\zeta$ and $\zeta^{\prime}$, there difference $\Delta=\zeta-\zeta^{\prime}$ is linear in $x$. Hence, $\Delta(x)=q x$ for some $q \in \boldsymbol{R}^{4}$, as $\Delta$ is continuous, and (3.7) is sufficiently general. This completes the proof of the lemma and, at the same time, completes the proof of Proposition 2.

As is well known, the second cohomology group $H^{2}(G, \boldsymbol{R})$ $=Z^{2}(G, \boldsymbol{R}) / B^{2}(G, \boldsymbol{R})$ is not trivial and, as a consequence, there exist numerous continuous projective representations of $G$ that are not induced by unitary representations [24]. Surprisingly enough, Proposition 2 tells us that any continuous projective representation of $G$ on $\mathfrak{S}_{0}$, implementing the automorphisms (3.1), is in fact induced by a continuous unitary representation $U_{\eta}$, given by (3.6) and (3.7).

From Stone's theorem we infer the existence of a spectral resolution

$$
U_{\eta}(x)=\int d E_{\eta}(p) e^{i p x}
$$

where $E_{\eta}$ is a finite projection-valued measure on $\boldsymbol{R}^{4}$, the dual of $G$. The physical interpretation of $\left(\Omega, E_{\eta}(B) \Omega\right)$ is that it predicts the probability of finding the measured value of the field momentum within the Borel set $B$, if the field is in the coherent state $E_{F}$ (so that $\eta$ is given by $(2 \mathrm{c})$ ). In view of (3.7) the scale of the momentum is determined only up to an arbitrary vector $q$ which can always be added. However, if the spectrum is bounded from below, $q$ may be adjusted so as to yield a momentum spectrum, acceptable from the physical point of view.

\section{The Spectral Condition}

Let $V_{a}$ denote the forward light cone with vertex $a \in \boldsymbol{R}^{4}$, that is the set $\left\{p+a: p^{2} \geqq 0, p_{0} \geqq 0\right\}$. The representation $U_{\eta}$ of $G$ as defined by (3.6) and (3.7) is said to satisfy the spectral condition if the following two statements are verified:

(I) $\operatorname{supp}\left(E_{\eta}\right) \subset V_{0}$.

(II) If $a \in V_{0}$ and $\operatorname{supp}\left(E_{\eta}\right) \subset V_{a}$, then $a=0$. 
As a necessary condition, $\operatorname{supp}(m) \subset V_{0}$ should hold for the measure $m$ defined by $\left(\Omega, U_{\eta}(x) \Omega\right)=\int d m(p)$ expipx. Evidently, $\left(\Omega, U_{\eta}(x) \Omega\right)$ $=\exp \left\{-\frac{1}{2}\|\xi(x)\|_{0}^{2}+i \zeta(x)\right\}$ and, introducing $\mu$ and $q$ from Lemma 3 , $\log \int d m(p) e^{i p x}=i q x+\int d \mu(\boldsymbol{k}) \frac{1+\boldsymbol{k}^{2}}{\boldsymbol{k}^{2}}\left[e^{i k x}-1-\frac{i k x}{\left(1+\boldsymbol{k}^{2}\right)^{\frac{1}{2}}}\right]_{k_{0}=|\boldsymbol{k}|}$

with a conditionally positive definite function [25] appearing on the right.

Lemma 4. Let $\mu$ be a finite positive Borel measure on $\boldsymbol{R}^{3}$, such that $\{0\}$ is a $\mu$-null set, and let the measure $m$ be defined by (4.1). For the support of $m$ to be contained in $V_{a}$ it is necessary and sufficient that

(i) $\int d \mu(\boldsymbol{k})\left(\frac{1+\boldsymbol{k}^{2}}{\boldsymbol{k}^{2}}\right)^{\frac{1}{2}}<\infty$,

(ii) $q-\int d \mu(\boldsymbol{k})\left(\frac{1+\boldsymbol{k}^{2}}{\boldsymbol{k}^{2}}\right)^{\frac{1}{2}}\left(\frac{k}{k_{0}}\right)_{k_{0}=|\boldsymbol{k}|} \in V_{a}$.

Proof. For brevity, $k=\{|\boldsymbol{k}|, \boldsymbol{k}\}$ always. Assume $\operatorname{supp}(m) \subset V_{a}$. By the uniqueness of the analytic continuation,

$$
\begin{aligned}
\int d \mu(k) \frac{1+\boldsymbol{k}^{2}}{\boldsymbol{k}^{2}}\left[e^{-k u}-1+\frac{k u}{\left(1+\boldsymbol{k}^{2}\right)^{\frac{1}{2}}}\right] & =(q-a) u+\log \int d m(p) e^{-(p-a) u} \\
& \leqq(q-a) u
\end{aligned}
$$

for any $u \in V_{0}$. Replacing $u$ by su with $s>0, u_{0}=1, \boldsymbol{u}=0$, and dividing by $s$ one gets $\int\left(f_{s}-g_{s}\right) d \mu \leqq q_{0}-a_{0}$, where

and

$$
f_{s}(\boldsymbol{k})=\left(1+\boldsymbol{k}^{2}\right)^{\frac{1}{2}} \int_{0}^{1} d t \int_{0}^{s t} d \lambda e^{-\lambda|\boldsymbol{k}|}
$$

$$
g_{s}(\boldsymbol{k})=\left[1+\left(1+\boldsymbol{k}^{2}\right)^{-\frac{1}{2}}\right]^{-1} \int_{0}^{|\boldsymbol{k}|} d t e^{-s t}
$$

define monotone increasing resp. decreasing sequences of non-negative bounded measurable functions, each of the integrals $\int f_{s} d \mu$ and $\int g_{s} d \mu$ being defined. Set

$$
f(k)=\left(\frac{1+k^{2}}{k^{2}}\right)^{\frac{1}{2}}
$$

Then, as $s$ tends to infinity, $f_{s}$ approaches $f$ pointwise except for $\boldsymbol{k}=0$. Since $\{0\}$ is a $\mu$-null set, $\lim f_{s}=f \mu$-almost everywhere and

$$
\int f_{s} d \mu \leqq q_{0}-a_{0}+\int g_{s} d \mu \leqq q_{0}-a_{0}+\int g_{0} d \mu \text {. }
$$


Hence, $I_{s}=\int f_{s} d \mu$, being a monotone increasing and bounded sequence, converges and its limit $I_{\infty}$ is the integral $\int f d \mu[26, \S 27$, Theorem B]. This proves the necessity of (i). Setting

$$
v=q-\int d \mu(\boldsymbol{k})\left(\frac{1+\boldsymbol{k}^{2}}{\boldsymbol{k}^{2}}\right)^{\frac{1}{2}} \frac{k}{|\boldsymbol{k}|}
$$

and applying the above inequality, we obtain

$$
(v-a) u \geqq-\int d \mu(\boldsymbol{k}) \frac{1+\boldsymbol{k}^{2}}{\boldsymbol{k}^{2}}\left(1-e^{-k u}\right) \geqq 0
$$

and thus $v \in V_{a}$, for $u \in V_{0}$ was arbitrary. This proves the necessity of (ii). Conversely, let (i) and (ii) be satisfied. Then the function

$$
K(z)=\exp \int d \mu(\boldsymbol{k}) \frac{1+\boldsymbol{k}^{2}}{\boldsymbol{k}^{2}}\left(e^{-z k u}-1\right), \quad z=s+i t, u \in V_{0},
$$

is holomorphic and of bounded modulus in the halfplane $s>0$. By a theorem of Schwartz [27, Chap. VI, Proposition 5], $K$ is the Laplace transform $\mathscr{L}(T)$ of a distribution $T \in \mathscr{D}^{\prime}$. On the other hand, $\mathscr{L}(T)(s)$ $=\int d m(p) \exp (-s(p-v) u)$ as a consequence of (4.1) and (4.2), showing that the support of $m$ must be contained in $S_{u}=\{p:(p-v) u \geqq 0\}$ and hence in the intersection $\cap S_{u \in V_{0}}=V_{v} \subset V_{a}$. This proves the sufficiency of (i) and (ii).

We are now prepared to formulate and prove the final result.

Proposition 3. The following statements are equivalent:

(3a) There exists a strongly continuous unitary representation of $G$ on $\mathfrak{H}_{0}$ that implements the automorphisms (3.1) and satisfies the spectral condition.

(3b) There exists a function $M(x)$ on $G$ such that $\lim _{x \rightarrow 0} M(x)=0$ and $|F(f-(x) f)| \leqq M(x)\|f\|_{1}$.

(3c) $F$ admits a unique decomposition $F^{(1)}+F^{(2)}$, where $F^{(1)}$ is translationally invariant and $F^{(2)}(f)=2 \operatorname{Im}(\eta, f)_{0}$ for some $\eta \in L_{1}$.

The representation of $G$, provided it exists, is uniquely determined and given by

$$
U_{\eta}(x)=e^{i \zeta(x)} U_{0}(x) W_{0}(\xi(x))
$$

where $\xi(x)=\left(I-V(x)^{*}\right) \eta$ and $\zeta(x)=\operatorname{Im}(\eta,(V(x)-I) \eta)_{0}$.

Proof. (3a) $\Rightarrow(3 \mathrm{c})$ : Combine Proposition 2, Lemma $3+4$, and notice that the condition (i) in Lemma 4 is equivalent to $\eta \in L_{1}$.

$(3 \mathrm{c}) \Rightarrow(3 \mathrm{~b})$ : Use $M(x)=2\left\|\left(I-V(x)^{*}\right) \eta\right\|_{-1}$ and (3.4).

$(3 \mathrm{~b}) \Rightarrow(3 \mathrm{c})$ : As $\|f\|_{1} \leqq\|f\|_{0}$, the existence of $\eta \in L_{2}$ follows from Proposition 2. Let $\xi(x)=\left(I-V(x)^{*}\right) \eta$ and let $\mu$ be defined as in Lemma 3 . 
For $\eta$ to be in $L_{1}$ it suffices to show that $|\boldsymbol{k}|^{-1}$ is $\mu$-integrable in some open neighborhood of $\boldsymbol{k}=0$. Now, $|F(f-(x) f)| \leqq M(x)\|f\|_{1}$ implies $\|\xi(x)\|_{-1}<M(x)$ for all $x \in G$, and thus

$$
\begin{aligned}
\int_{|\boldsymbol{k}|<1} d \mu(\boldsymbol{k})|\boldsymbol{k}|^{-1}<\int d \mu(\boldsymbol{k})\left(\frac{1+\boldsymbol{k}^{2}}{\boldsymbol{k}^{2}}\right)^{\frac{3}{2}} & \left|e^{i|\boldsymbol{k}| \pi}-1\right|^{2} \\
& =\|\xi(a)\|_{-1}^{2}<M(a)^{2}
\end{aligned}
$$

for $a=\{\pi, 0,0,0\}$.

(3c) $\Rightarrow(3 \mathrm{a})$ : Given $\eta \in L_{1}$, let $U_{\eta}$ be defined by (4.3). Our previous discussion has shown that $U_{\eta}$ is a strongly continuous unitary representation of $G$ implementing (3.1). Let $\mu$ and $q$ be defined by Lemma 3. Then

$$
\int d \mu(\boldsymbol{k})\left(\frac{1+\boldsymbol{k}^{2}}{\boldsymbol{k}^{2}}\right)^{\frac{1}{2}}=\|\eta\|_{1}^{2}<\infty
$$

verifies that the condition (i) of Lemma 4 is fulfilled. Further, let $v$ be defined by (4.2). If $v=0$ or, equivalently, if $\zeta(x)=\operatorname{Im}(\eta,(V(x)-\mathrm{I}) \eta)_{0}$, then the condition (ii) is satisfied for $a=0$, and $\operatorname{supp}(m) \subset V_{0}$ as a consequence of that lemma, where $\int d m(p) \operatorname{expipx}=\left(\Omega, U_{\eta}(x) \Omega\right)$. Suppose, there is some $a \in V_{0}$ such that $\operatorname{supp}(m) \subset V_{a}$. Then $a=0$ from $v=0$ and Lemma 4 (b). Thus, $U_{\eta}$ satisfies the spectrum condition at least in the state given by the vector $\Omega$. It remains to show that the same assertion holds for a sufficient large set of vector states. Given $\delta \in L_{0}$ we obtain

$$
W_{0}(\delta)^{*} U_{\eta}(x) W_{0}(\delta)=U_{\eta+\delta}(x)
$$

as a result of a simple calculation. Setting $\Phi_{\delta}=W_{0}(\delta) \Omega$, we learn that $\left(\Phi_{\delta}, U_{\eta}(x) \Phi_{\delta}\right)=\left(\Omega, U_{\eta+\delta}(x) \Omega\right)$ again has the correct spectrum, since $\eta+\delta \in L_{1}$. So the properties (I) and (II) are verified for the spectral measure $E_{\eta}$ of $U_{\eta}(x)$, as $\left\{\Phi_{\delta}: \delta \in L_{0}\right\}$ provides a total set of vectors in $\mathfrak{H}_{0}$. This completes the proof.

Several remarks are in order.

(1) Since the quotient $L_{2} / L_{1}$ is not trivial, one may construct numerous Weyl systems $\left(\mathfrak{H}_{0}, W_{F}, \Omega\right)$ that, although admitting a momentum operator, do not satisfy the spectrum condition. In these cases the energy spectrum necessarily extends to $-\infty$. It is emphasized that such systems arise in external current models where the Fourier transform $j(k)$ of the current behaves as singular as $|\boldsymbol{k}|^{\varepsilon-2}, 0<\varepsilon \leqq \frac{1}{2}$, at the vertex of the light cone.

(2) Disregarding translationally invariant functionals $F^{(1)}$, we have an one-to-one correspondence between the elements of $L_{1} / L_{0}$ and certain sectors that admit a momentum operator with physical spectrum. The zero vector in $L_{1} / L_{0}$ corresponds to the vacuum sector, whereas all non- 
trivial vectors correspond to so-called infrared sectors. This essentially confirms some of the results obtained by Kibble [9].

(3) Nowhere have we assumed that $\zeta(x)$ is differentiable and the partial derivatives of $\xi(x)$ are likely to be something outside of $L_{0}$. Apparently, these aspects crucially depend on the high frequency behavior of $\eta$ and, so far, were irrelevant for our discussion. However, let us introduce the unbounded selfadjoint generators of the time translations: $V\left(x_{0}\right)=\exp i h x_{0}, U_{\eta}\left(x_{0}\right)=\exp i H_{\eta} x_{0}$. If $\eta=0, H_{\eta}$ becomes the hamiltonian for the Fock system. There is no problem with first order derivatives if $h \eta \in L_{-1}$. In particular,

$$
H_{\eta} \supset H_{0}+A\{i h \eta\}+(\eta, h \eta)_{0} I
$$

which follows from (4.3) and $W_{0}(t \xi)=\exp i t A\{\xi\}$. Symmetric operators of the form of the right hand side have often been the starting point for a construction of $U_{n}\left(x_{0}\right)$. Notice, however, that the domain of definition shrinks for that operator as the high energy behavior of $\eta$ becomes worse. In cases where the self-energy term $(\eta, h \eta)_{0}$ is infinite, the three operators $H_{\eta}, H_{0}, A\{i h \eta\}$ have no common vector of definition except the zero vector.

(4) A space rotation mixes the infrared sectors as can be readily seen. Consider a state $E_{F}$ with $F(f)=2 \operatorname{Im}(\eta, f)_{0}, \eta \in L_{1}$, and let $V(R)$ be the strongly continuous unitary representation of the rotation group on $L_{1}$. For $\eta$ and $V(R)^{*} \eta$ to define the same sector it is necessary and sufficient that $\left(I-V(R)^{*}\right) \eta \in L_{0}$. Suppose this holds for any rotation $R$. Let $v$ be the normalized Haar measure on the rotation group. Since $V(R)$ does not contain the trivial representation, $\int d v(R) V(R)^{*} \eta=0$ and thus $\eta \in L_{0}$, saying that $E_{F}$ is in the vacuum sector. Therefore, the question "What is the angular momentum carried by the infrared radiation?" does not make sense. Apparently, it requires a separation of the charged system from its radiation field which is impossible in principle. Of course, it is puzzling that the angular momentum should not be defined, although the ordinary momentum is. Further, it is evident that, if all proper Lorentz transformations can be unitarily implemented, then $E_{F}$ again is in the vacuum sector, since any rotation may be written as a product of three proper Lorentz transformations.

(5) The infrared correction factor, which is used in scattering theory to account for unobserved low frequency radiation, is simply related to the spectral resolution of $\left(\Omega, U_{\eta}(x) \Omega\right)$. Suppose $n$ incoming and $m$ outgoing particles take part in a scattering process. Let their momenta be $-p_{1}, \ldots,-p_{n}, p_{n+1}, \ldots, p_{n+m}$ relative to the center-of-mass system and let their charges be $-e_{1}, \ldots,-e_{n}, e_{n+1}, \ldots, e_{n+m}$, subject to the restrictions $\sum p_{\alpha}=0$ and $\sum e_{\alpha}=0$. With no soft radiation incoming, the soft photon emission will be almost coherent and is well approximated 
by a state $E_{F}$, where $F(f)=2 \operatorname{Im}(\eta, f)_{0}=\int d x \int d y J_{s}^{\mu}(x) D(x-y) \varphi_{\mu}(y)$ and $J_{s}$ is the low frequency part of the convection current $J$ produced by the classical point particles:

$$
J^{\mu}(x)=\sum_{\alpha} e_{\alpha} p_{\alpha}^{\mu} \int_{0}^{\infty} d \lambda \delta\left(x-\lambda p_{\alpha}\right) .
$$

Though the correct current should take into account the quantummechanical description of the charged particles in question, considerable deviations from the simple expression (4.6) are not to be expected in the low frequency range. It is easily checked that $\eta \in L_{1}$, but $\eta \notin L_{0}$. The infrared correction factor is then defined to be the probability $\left(\Omega, E_{\eta}(B) \Omega\right)$ for the radiation $E_{F}$ to have a momentum confined to the region $B$, the precise shape of $B$ being given by the experimental arrangement and the resolution of the counters. The general formula

$$
\left(\Omega, U_{\eta}(x) \Omega\right)=\exp (\eta,(V(x)-I) \eta)_{0},
$$

valid for $\eta \in L_{1}$, can be used to yield

$$
\begin{aligned}
&\left(\Omega, E_{\eta}(B) \Omega\right)=\frac{1}{(2 \pi)^{4}} \int_{B} d p \int_{R^{4}} d x \\
& \cdot \exp \left\{\frac{1}{(2 \pi)^{3}} \int_{|\boldsymbol{k}| \leqq s} \frac{d \boldsymbol{k}}{2|\boldsymbol{k}|}\left[\left(\mathrm{e}^{i k x}-1\right) \sum_{\alpha, \beta} \mathrm{e}_{\alpha} \mathrm{e}_{\beta} \frac{-p_{\alpha} p_{\beta}}{\left(p_{\alpha} k\right)\left(p_{\beta} k\right)}\right]_{k_{0}=|\boldsymbol{k}|}-i p x\right\} .
\end{aligned}
$$

Here, $s$ is a suitable low frequency cutoff. This expression is widely known [1].

Acknowledgements. The author is greatly indebted to J. E. Roberts, M. Rinke, and V. Enß for many discussions and helpful comments. He is also grateful to Prof. R. Haag and Prof. K. Symanzik for having organized a stimulating seminar on infrared problems.

\section{References}

1. Jauch, J. M., Rohrlich, F.: Helv. Phys. Acta 27, 613 (1954).

2. Glauber, R.: Phys. Rev. 130, 2529 (1963); 131, 2766 (1963).

3. Klauder, J. R., Sudarshan, E. C. G.: Fundamenatls of quantum optics. New York: Benjamin 1968.

4. Chung, V.: Phys. Rev. 140, B 1110, (1965).

5. Greco, M., Rossi, G.: Nuovo Cimento 50 A, 168 (1967).

6. Storrow, J. K.: Nuovo Cimento 54 A, 15 (1968).

7. Kibble, T. W. B.: Phys. Rev. 173, 1527; 174, 1882; 175, 1624 (1968).

8. Glauber, R. J.: Phys. Rev. 84, 395 (1951).

9. Kibble, T. W. B.: J. Math. Phys. 9, 315 (1968).

10. Bloch, F., Nordsieck, A.: Phys. Rev. 52, 54 (1937).

11. Pauli, W., Fierz, M.: Nuovo Cimento 15, 167 (1938).

12. Blanchard, Ph.: Commun. Math. Phys. 15, 156 (1969). 
13. Klauder, J. K., McKenna, J., Woods, E. J.: J. Math. Phys. 7, 882 (1966).

14. - J. Math. Phys. 11, 609 (1970).

15. Streater, R. F.: Lectures at the Varenna Summer School 1968.

16. Roberts, J. E., Roepstorff, G.: Commun. Math. Phys. 11, 321 (1969).

17. Segal, I. E.: Mat. Fys. Medd. Danske Vid. Selsk. 31, no. 12 (1959).

18. - Canad. J. Math. 13, 1 (1961).

19. Shale, D.: Trans. Am. Math. Soc. 103, 149 (1962).

20. Kastler, D.: Commun. Math. Phys. 1, 14 (1965) and therein quoted literature.

21. Manuceau, J.: Ann. Inst. Henri Poincaré 8, 117 (1968).

22. - Ann. Inst. Henri Poincaré 8, 139 (1968).

23. Araki, H.: Publ. RIMS 5, 361 (1970).

24. Bargmann, V.: Ann. Math. 59, 1 (1954), Sec. 6a.

25. Gel'fand, I. M., Vilenkin, N. Ya.: Generalized functions, Vol. 4, Chap. II, Sec. 4. New York-London: Academic Press 1964.

26. Halmos, P. R.: Measure theory. Princeton: Van Nostrand 1950.

27. Schwartz, L.: Méthodes mathematiques pour les sciences physique. Paris: Hermann 1961.

G. Roepstorff

II. Institut für Theoretische Physik der Universität

D-2000 Hamburg 50, Luruper Chaussee 149 